\title{
Signatures of landscape and captivity in the gut microbiota of Southern Hairy-nosed Wombats (Lasiorhinus latifrons)
}

\author{
Raphael Eisenhofer ${ }^{1,2^{*}}$ (D), Kristofer M. Helgen ${ }^{3,4}$ and David Taggart ${ }^{5,6}$
}

\begin{abstract}
Background: Herbivorous mammals co-opt microbes to derive energy and nutrients from diets that are recalcitrant to host enzymes. Recent research has found that captive management-an important conservation tool for many species - can alter the gut microbiota of mammals. Such changes could negatively impact the ability of herbivorous mammals to derive energy from their native diets, and ultimately reduce host fitness. To date, nothing is known of how captivity influences the gut microbiota of the Southern Hairy-nosed Wombat (SHNW), a large herbivorous marsupial that inhabits South Australia. Here, using $16 \mathrm{~S}$ rRNA gene sequencing, we characterized the faecal microbiota of SHNWs in captivity and from three wild populations, two from degraded habitats and one from an intact native grass habitat.

Results: We found that captive SHNWs had gut microbiota that were compositionally different and less diverse compared to wild SHNWs. There were major differences in gut microbiota community membership between captive and wild animals, both in statistically significant changes in relative abundance of microbes, and in the presence/absence of microbes. We also observed differences in microbial composition between wild populations, with the largest difference associated with native vs. degraded habitat.

Conclusions: These results suggest that captivity has a major impact on the gut microbiota of SHNWs, and that different wild populations harbour distinct microbial compositions. Such findings warrant further work to determine what impacts these changes have on the fitness of SHNWs, and whether they could be manipulated to improve future management of the species.
\end{abstract}

\section{Background}

There is increasing recognition that host-associated communities of microorganisms (microbiota) play key roles in animal health and should be a considered factor in wildlife management practices [1]. The gut microbiota has been demonstrated to influence host health through interactions with the immune system, behaviour, digestion, and essential nutrient synthesis (reviewed in [2-4]). For instance, herbivorous mammals can harbor microbes

\footnotetext{
* Correspondence: Raph.eisenhofer@gmail.com

'School of Biological Sciences, University of Adelaide, Adelaide, South

Australia, Australia

${ }^{2}$ Australian Research Council Centre for Australian Biodiversity and Heritage,

University of Adelaide, Adelaide, South Australia, Australia

Full list of author information is available at the end of the article
}

that detoxify plant defence compounds [5], increasing their dietary niche breadth. Additionally, it has long been understood that the fermentation of plant compounds by the gut microbiota, for example within the complex compartmentalized digestive systems of foregut fermenters like ruminant mammals, can make substantial contributions to the daily energy requirements of herbivores. It has been estimated that short-chain fatty acids (SCFA) produced by the gut microbiota of Southern Hairy-nosed Wombats (SHNWs), a hindgut fermenter, account for $>60 \%$ of the daily energy requirement of the host [6]. Loss or disruptions of these host-associated microbiota and functions could therefore have major implications for the health and fitness of animals.

(c) The Author(s). 2021 Open Access This article is licensed under a Creative Commons Attribution 4.0 International License, which permits use, sharing, adaptation, distribution and reproduction in any medium or format, as long as you give appropriate credit to the original author(s) and the source, provide a link to the Creative Commons licence, and indicate if changes were made. The images or other third party material in this article are included in the article's Creative Commons licence, unless indicated otherwise in a credit line to the material. If material is not included in the article's Creative Commons licence and your intended use is not permitted by statutory regulation or exceeds the permitted use, you will need to obtain permission directly from the copyright holder. To view a copy of this licence, visit http://creativecommons.org/licenses/by/4.0/. 
Captive breeding is an important wildlife management tool for many species. However, captivity can drastically modify the natural mammalian gut microbiota through various factors including changes in diet and antibiotic treatment [7-10] (reviewed in [1]). Diet is known to have a major influence on the gut microbiota of mammals [11]. Sonnenburg et al. [12] demonstrated that a change in diet can shift gut microbiota diversity and composition in laboratory mice (Mus musculus) and that, while these shifts were reversible in a single generation by dietary changes, they could not be restored by dietary intervention alone after multiple generations. Such microbial 'extinctions' were only reversible by reintroduction of both the missing microbes and diet. MartínezMota et al. [13] showed that white-throated woodrats (Neotoma albigula) brought into captivity and fed their natural diet retained more of their native microbiota when compared to animals fed an artificial diet. Determining which mammals experience effects of captivity on their gut microbiota is an important first step in developing management practices to retain native gut microbial diversity.

Animals of the same species living in geographically different populations can also have distinct gut microbiota compositions, which may be locally adaptive. Black howler monkeys (Alouatta pigra) were found to have habitat-specific gut microbiota signatures [14]. Monkeys living in degraded forests harboured lower gut microbial diversity and fewer microbes capable of producing butyrate, a SCFA energy source for the host. Similar trends were found in another study on two populations of Udzungwa red colobus monkeys (Procolobus gordonorum) [15]. The gut microbiota of animals living within the same population can also differ substantially. Koalas (Phascolarctos cinereus) from the same population were found to have distinct gut microbiota signatures associated with the consumption of different Eucalyptus species [16], and emerging evidence suggests that the gut microbiota of koalas can influence diet selection [17]. Identifying inter- and intra-population gut microbiota signatures could therefore yield important information from which to make wildlife management decisions, such as coordination/matching of individuals and locations to maximise translocation success.

To date, little is known about the gut microbiota of the Southern Hairy-nosed Wombat (SHNW) (Lasiorhinus latifrons). The SHNW is a large, sedentary, semifossorial marsupial herbivore, found in semiarid grassland habitats across southern South Australia, west of the Murray River to the south-eastern corner of Western Australia [18-20]. It is a grazer and a hindgut fermenter, with microbially-facilitated digestion occurring especially in an expansive colon $[6,21,22]$. Animals are subjected to annual extremes in water and nutrient availability, with limited access to water or food during summer and autumn months. Feed becomes prevalent after rainfall, which typically occurs in winter and spring, unless the region is in drought $[18,23,24]$. The species displays significant physiological and behavioural adaptations for water and energy conservation in this harsh environment, including a low basal metabolic rate, ever-growing cheek teeth well-adapted to mechanically reducing food into small particulates, long gut retention times, production of dry faecal pellets, and a relatively inactive, burrowing lifestyle [22-27]. The home range of this species is very small $(2-4 \mathrm{ha})$ for an animal of their size and centered around their preferred warrens [28]. In intact habitats, animals feed mostly on native perennial grasses - Austrostipa spp., forbs and chenopods (saltbush - Atriplex, Enchylaena and Rhagodia spp., and bluebush - Maireana spp.) and it is recognized as an important ecosystem engineer [24, 29]. When native vegetation is unavailable, as in degraded habitats, a variety of introduced weed species are eaten [24]. The species is also of conservation concern, with a formerly broad geographic range in southern Australia fragmented by agriculture, livestock production, and other land-use changes, and is currently listed by the IUCN as "Near-threatened." It is also closely related to the critically endangered northern hairy-nosed wombat (Lasiorhinus krefftii [30]). Nothing is known about whether habitat type influences the gut microbiota of SHNWs, or whether different populations of SHNWs have distinct gut microbiota.

To better understand whether factors such as captivity or habitat type influence the gut microbiota of SHNWs, we collected and characterised the microbial composition of fresh faecal samples from one captive and three wild populations in South Australia (two of which represent degraded, and one intact habitat). We hypothesised that 1) captivity results in both the reduction of diversity and a substantial change in gut microbial composition, and 2) different wild populations harbour distinct gut microbial compositions.

\section{Methods \\ Study sites and habitat \\ Captive}

Captive SHNWs accessed for this study were held by an experienced wildlife carer in the Adelaide Hills, approximately $10 \mathrm{~km}$ south-west of Adelaide, South Australia. These were rescue animals brought in from across the state, with most being hand-reared from a young age. The animals were housed in separate, custom-built enclosures containing burrows, and fed Barastoc Complete Performer (primarily cooked and flaked barley and lupins, lucerne and cereal chaff). The diet was bolstered in the wintertime with sunflower, oats, carrots, and sweet potato. Animals had access to water ad-lib. 


\section{Degraded habitat - Kooloola Station and Brookfield Conservation Park}

Wild SHNWs from degraded habitat were located at Kooloola Station and Brookfield Conservation Park (Fig. 1). Both sites were historic sheep grazing properties and are located approximately $2.5 \mathrm{~h}$ drive north east of Adelaide, in South Australia's Murraylands region. The annual rainfall in this area is $\sim 270 \mathrm{~mm}$ (Bureau of Meteorology - BOM, 2018). The area consists of remnant mallee eucalypt woodland with a chenopod and grassland understory composed of a variety of introduced weeds, native grasses and chenopods. The diet of wombats in this area is made up largely of introduced weed species including thread iris (Moraea setifolia), wards weed (Carrichtera annua) and burr medic (Medicago minima), along with remnant chenopods and a few native grass species [31]. Except immediately after rain, no free water is available at these sites.

\section{Intact habitat - Wonga Station}

Wild SHNWs from intact habitat were located at Wonga Station, a sheep grazing property of $\sim 130,000$ acres, located about $3.5 \mathrm{~h}$ drive north east of Adelaide, in South Australia's Murraylands region (Fig. 1). This area receives $\sim 290 \mathrm{~mm}$ of rainfall annually (BOM, 2018). The vegetation on this property is predominantly composed of intact, native grassland habitat interspersed with remnant mallee eucalypt woodland. Active weed management is undertaken at this site. Native grasses and forbs, the dominant component of wombat diet in this region, flourish at this site and include many Stipia, Hyalosperma, Silene, Rytidiosperma and Sida spp., and a wide variety of Chenopodiaceae (saltbush - Atriplex, Enchylaena and Rhagodia spp., and bluebush - Maireana spp.) among others [31]. Except immediately after rain, no free water is available at this site.

\section{Sample collection}

Once faeces have been deposited by an animal, the microbes inside can continue to grow and distort the true representation of the microbial community as it was inside the host [32]. To counteract this potentially confounding variable, faecal samples are best collected fresh and preserved. Freezing is a commonly used preservative method, but is difficult to use in a fieldwork context. Instead, we opted for preservation of faecal samples by immersion in 95\% ethanol, which has been demonstrated to reliably preserve faecal microbial community composition at room temperature [32]. We collected samples in the evening/early morning when SHNWs are most active, offering the best opportunity to collect fresh faecal samples.

The entrance and soil mound area around SHNW warrens were searched for fresh faecal pellets. Old pellets and fresh pellets were qualitatively distinguished by gently squeezing with a freshly gloved hand. Once found, fresh pellets were placed on a piece of aluminium foil and cut in half using the cutting edge of a spatula. The pellet cores were extracted using the opposite end of the spatula and placed in $2 \mathrm{~mL}$ plastic screw-top tubes prefilled with $1.5 \mathrm{~mL}$ of $95 \%$ ethanol, before being shaken vigorously to ensure mixing of sample with ethanol. Gloves were changed between samples, and spatulas were decontaminated using $5 \%$ bleach (sodium hypochlorite) followed by an ethanol wash.

Samples from Kooloola Station ( $n=21$ from 11 warrens) were collected in the evening of 13 March 2019; Brookfield samples ( $n=17$ from 8 warrens) were

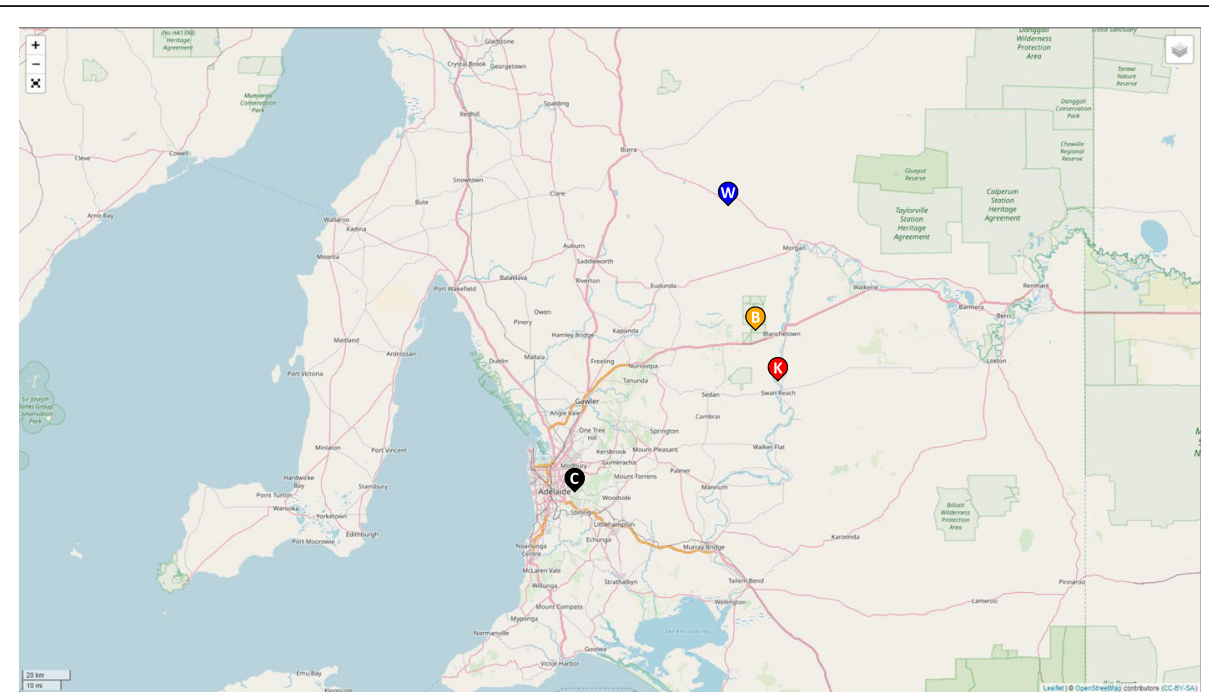

Fig. 1 Map of study sites in South Australia. C = captive population $(n=21), \mathrm{K}=$ Kooloola Station, degraded habitat $(n=21), \mathrm{B}=$ Brookfield Conservation Park, degraded habitat $(n=17)$, and $\mathrm{W}=$ Wonga Station, intact habitat $(n=23)$ 
collected in the morning of 14 March 2019 (mean overnight temperature of $7.2{ }^{\circ} \mathrm{C}$; Eudunda weather station, Bureau of Meteorology). Samples from Wonga Station ( $n=23$ from 11 warrens) were collected overnight and into the morning of 19 June 2019 (mean overnight temperature of $1{ }^{\circ} \mathrm{C}$; Eudunda weather station, Bureau of Meteorology). For detailed maps of the sample sites, see https://github.com/EisenRa/2020_SHNW_Faecal_16S/ tree/main/Site_maps. Scat samples from captive animals $(n=21)$ were collected between 13 May 2019 and 5 June 2019 , with some individuals being sampled up to three different time points (multiple samples from the same individual were not pooled, but processed separately and sample names suffixed with: a, b, c). A soil sample from each wild study site was also collected for analysis.

\section{DNA extraction}

All DNA extractions were performed in freshly decontaminated Perspex hoods in a pre-PCR laboratory to prevent contamination with amplicons [33]. DNA was extracted using the QIAGEN DNeasy PowerSoil kit (formerly MO BIO PowerSoil) according to the manufacturer's protocol. To reduce contamination, all buffers and tubes needed for the various steps were aliquoted prior to opening any sample tubes. Faecal samples were centrifuged at 10,000 $\mathrm{g}$ for $5 \mathrm{~min}$ before pouring the ethanol off. Because SHNW faeces are very dry, only $\sim 150$ mg from each sample was used. To minimise batch effects samples in extraction groups were randomized, and to account for laboratory related contamination extraction blank controls from each extraction group was included and carried through to DNA sequencing [33].

\section{Amplicon library preparation, quantification, and DNA sequencing}

All samples were PCR-amplified and uniquely barcoded for High-Throughput Sequencing (HTS) using primers targeting the V4 region of the bacterial 16S rRNA gene [34]. We used forward primer: 515F (AATGATACGG CGACCACCGAGATCTACACTATGGTAATTGTGTGCCAGCMGCCGCGGTAA) and barcoded reverse primer 806R (CAAGCAGAAGACGGCAT-ACGA GATnnnnnnnnnnnnAGTCAGTCAGCCGGAC-

TACHVGGGTW TCTAAT) - the 12 n's represent unique barcode sequences. The PCR reactions were prepared in a pre-PCR laboratory in a $5 \%$ bleached-cleaned and UV irradiated hood. Single reactions [35] of $2.5 \mu \mathrm{L}$ X10 HiFi buffer, $0.1 \mu \mathrm{L}$ Platinum $^{\mathrm{mm}}$ Taq DNA Polymerase (ThermoFisher), $19.2 \mu \mathrm{L}$ dH2O, $0.2 \mu \mathrm{L} 100 \mathrm{mM}$ dNTP mix, $0.5 \mu \mathrm{L}$ each of $10 \mu \mathrm{M}$ forward and reverse primer and $1 \mu \mathrm{L}$ DNA. DNA was amplified using an initial denaturation at $94{ }^{\circ} \mathrm{C}$ for $3 \mathrm{~min}$, followed by 35 cycles of denaturation at $94{ }^{\circ} \mathrm{C}$ for $45 \mathrm{~s}$, annealing at $50{ }^{\circ} \mathrm{C}$ for 1 min, elongation at $68{ }^{\circ} \mathrm{C}$ for $90 \mathrm{~s}$, with final adenylation for $10 \mathrm{~min}$ at $68{ }^{\circ} \mathrm{C}$, in line with the Earth Microbiome Protocol [36].

Gel electrophoresis was carried out for each PCR reaction on a $3.5 \%$ agarose gel to ensure the samples contained library constructs of the desired length ( $390 \mathrm{bp})$. For each sample, $1 \mu \mathrm{L}$ amplified DNA was mixed into

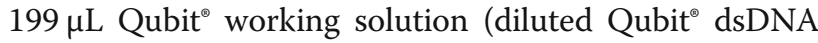
HS Reagent 1:200 in Qubit dsDNA HS Buffer) and quantified using a Qubit $^{\circ}$ 2.0 Fluorometer. Samples were pooled equimolar and cleaned using AxyPrep ${ }^{\text {Tw }}$ (Axygen) following the manufacturer's instructions. Because negative controls contained little DNA, they were pooled separately and spiked into the final pool at a flat volume [33]. The final pool was quantified and quality checked using an Agilent TapeStation. DNA sequencing was performed on an Illumina MiSeq (v2, $2 \times 150 \mathrm{bp}$ ) at SAHM RI (South Australian Health and Medical Research Institute).

\section{Data processing and statistical analyses}

DNA sequencing data were processed and analysed using QIIME2 v2020.2 [37]. An interactive Jupyter notebook containing all the QIIME2 code used is available (https://github.com/EisenRa/2020_SHNW_Faecal_16S/ blob/main/SHNW_Gut_16S_2019.ipynb). For captive animals that had multiple collections we randomly selected one sample for further downstream processing (see the Jupyter notebook for details). Forward reads (R1) were imported into the QIIME2 and denoised into amplicon sequence variants (ASVs) using the deblur [38] plugin with a trim length of $150 \mathrm{bp}$. Representative sequences were assigned taxonomy using the QIIME2 feature-classifier plugin (naive bayesian approach) on the pre-trained SILVA [39] 132 V4 region classifier [40]. A phylogenetic tree was created using the SATé-enabled phylogenetic placement (SEPP) technique [41] within the fragment-insertion QIIME2 plugin. Alpha diversity (Observed OTUs and Faith's Phylogenetic diversity [42]) and Beta diversity (weighted [43] and unweighted [44] UniFrac metrics) were calculated using the QIIME2 diversity plugin with a rarefaction depth of 36,346 sequences per sample (see https://github.com/EisenRa/2020_SHNW_ Faecal_16S/blob/main/SHNW_Gut_16S_2019.ipynb for the rarefaction curve). Tests for differentially abundant microbes between populations were performed using ANCOM [45]. SINA (SILVA Incremental Aligner) [46] was used to search specific ASVs against the SILVA 138 database. We used SourceTracker2 (https://github.com/biota/ sourcetracker2) [47] to assess whether microbes from the soil were a source for faecal samples. PCoA, Alpha diversity, and Venn diagrams were constructed using phyloseq [48] and ggplot2 [49] in R Studio [50], see the Rmarkdown file for details (https://github.com/EisenRa/2020_SHNW_ Faecal_16S/blob/main/Figures.rmd). Maps were generated 
using Geoplaner (www.geoplaner.com) with data from OpenStreetMaps (wiki.openstreetmap.org).

\section{Removal of outlier samples}

We observed 7 samples collected from the 4 Easternmost warrens from Brookfield Conservation Park that appeared to be outliers in our dataset. PCoA of unweighted UniFrac distances clearly separated these samples from other wild and captive samples (SI Figure 1). Additionally, the diversity in these outlier samples was higher than other Brookfield samples (mean of $1256 \mathrm{ob}-$ served ASVs vs. 828). The taxonomic composition of these outlier samples was also substantially different to other samples (SI Figure 2). An interactive view at different taxonomic levels can be observed by dragging SI File 2 into the https://view.qiime2.org/ webpage. The most stark difference between these outlier samples and the other samples in the dataset is the loss of Spirochaetes, which was present in all other wild samples with a mean relative abundance of $11.6 \%$ and captive samples at $5.1 \%$. Additionally, these samples did not have any $I z i-$ maplasmataceae (average $5.4 \%$ in the other wild samples). A possible cause for these differences could be taphonomy-perhaps these faecal samples were older than they seemed, which could have resulted in a shift of observed community composition. Alternatively, there could be true biological differences between these samples that we were unable to determine due to our noninvasive sampling technique (host health or disease, etc.). Due to these samples being so different to other wild samples (greater than between population differences or captive vs. wild) we chose to exclude these outlier samples from subsequent analyses.

\section{Frequency-based filtering to mitigate cross-sample contamination}

Samples undergoing DNA extraction and library preparation in the same batch can be affected by cross-sample contamination, whereby cells (from DNA extraction) or DNA (from DNA extraction and/or library preparation) can cross from a sample to another $[33,51]$. Such crosssample contamination can therefore yield false-positive detection of microbes within samples, with samples of higher microbial biomass being more likely to spillover [51]. Because we randomized sample order within DNA extractions and library preparations (to reduce batch effects), there is a possibility of spillover between faecal samples from different populations. This would confound attempts at identifying ASVs that are unique to a particular population (i.e. Figs. 3b, 5). To try and mitigate the effect of cross-sample contamination for population-specific ASV analysis, we applied a conservative frequency-based filtering approach, whereby population-specific ASV tables were filtered to remove
ASVs with a relative abundance of $<0.00015 \%$ ). This threshold was based on the frequency of the most abundant ASV in the dataset (3c44df3672100a011a334b67eea24366), which was found in all wild samples with a total frequency of 278,742 reads (5.8\% of total reads in wild samples). In contrast, this ASV was only found in the captive-only table with a total frequency of 131 reads ( $0.0001 \%$ of the captive table abundance). Assuming that the most abundant ASV would result in the greatest number spillover events, setting a threshold based on this ASV $(0.00015 \%)$ would account for the spillover of less abundant ASVs. See the interactive Jupyter notebook for more details and the exact code ran (https:// github.com/EisenRa/2020_SHNW_Faecal_16S/blob/ main/SHNW_Gut_16S_2019.ipynb). The issue of crosssamples contamination in microbiome studies clearly deserves further research.

\section{Results}

DNA sequencing of the 97 samples resulted in 13,545, 820 reads (mean of 139,647), which were denoised using deblur into 8483 amplicon sequence variants (ASVs).

\section{Captivity influences SHNW gut microbiota diversity and composition}

Microbial diversity was higher in wombat scats collected from wild populations compared to those sourced from captivity (Fig. 2a \& b). All three wild populations had statistically significantly higher microbial phylogenetic diversity versus the captive population (Fig. 2a; KruskalWallis tests of Faith's phylogenetic diversity $p$-values < 0.001 SI Table 1). This finding was mirrored with microbial richness, with the exception of captive versus Wonga (Fig. 2b; Kruskal-Wallis tests of richness $p$-values $<0.05$ SI Table 2). Analysis of microbial composition via principal coordinates analysis ( $\mathrm{PCoA})$ of unweighted UniFrac distances showed clustering of samples by captivity status (Fig. 2c \& d). Captive samples were separated from wild samples across the first principal coordinate, which explained $31 \%$ of the variation, and these differences in microbial composition were statistically significant (PERMANOVA of unweighted UniFrac distances $p$-value $=0.001$, pseudo-F 26.7). This result was also found when using the weighted (by abundance) Unifrac metric (SI Figure 3; PERMANOVA of weighted UniFrac distances $p$-value $=0.001$, pseudo-F 19.4).

The microbial phyla with the greatest relative abundance in SHNW faecal samples were Firmicutes (39.5 and $64.4 \%$ relative abundance for wild and captive, respectively), Bacteroidetes (29.7\% wild, $13.3 \%$ captive), Tenericutes (12.1\% wild, $10.3 \%$ captive), and Spirochaetes (11.6\% wild, 5.1\% captive) (SI File 1). The Firmicutes:Bacteroidetes ratio was substantially higher in captive animals (mean 4.8:1) compared to wild (Kooloola \& Brookfield $=1.7: 1$, 


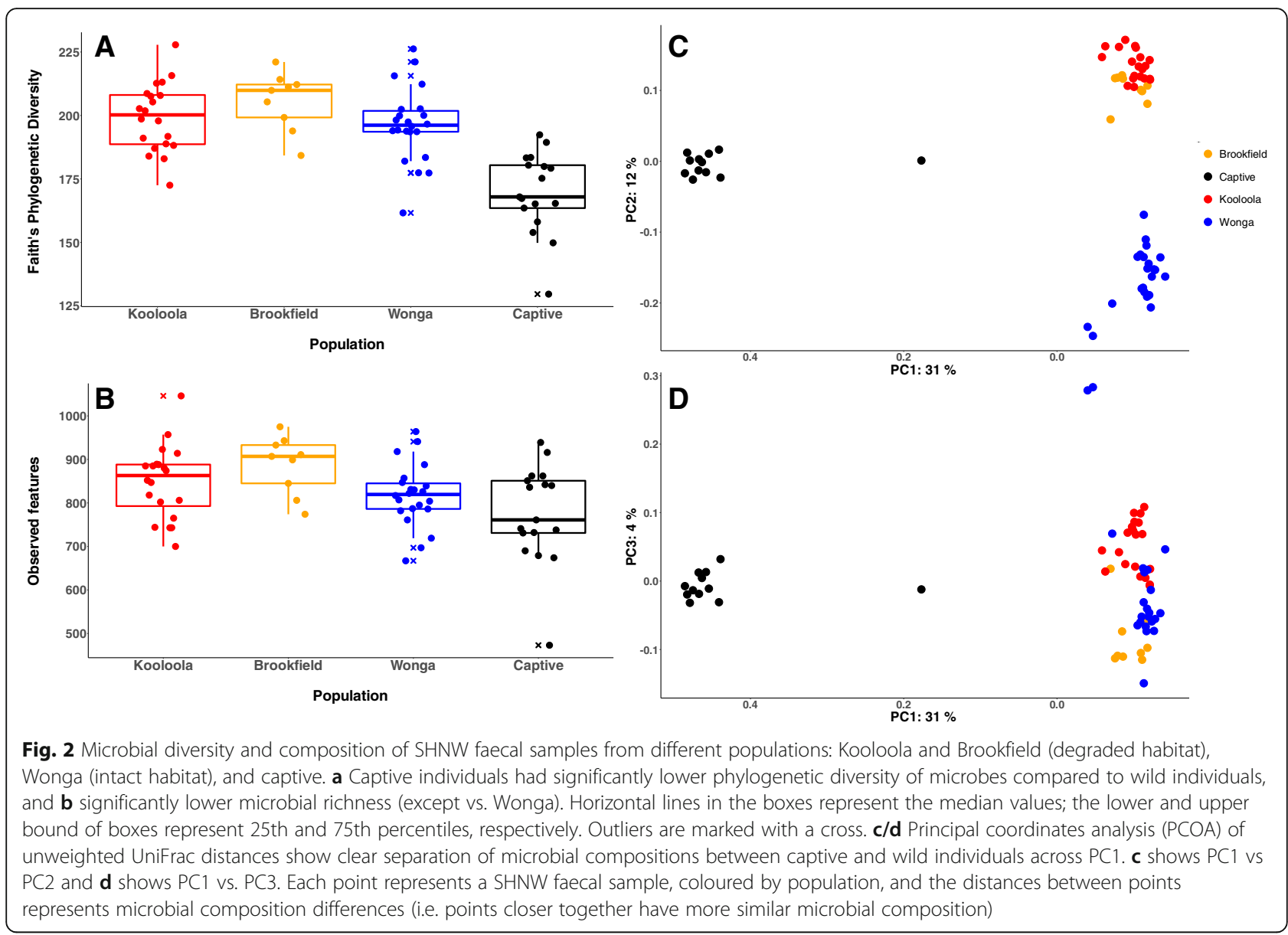

Wonga $=0.8: 1$ ). Twenty five microbial families accounted for $\sim 90 \%$ of the faecal microbiota, with the three most abundant families being Ruminococcaceae (20.9\% wild, $33.1 \%$ captive), Spirochaetaceae ( $11.5 \%$ wild, $5.1 \%$ captive), Rikenellaceae (12.2\% wild, 5.1\% captive), and Christensenellaceae (5.5\% wild, $8.5 \%$ captive) (Fig. 3 and SI File 1). Interestingly, some families were only detected in wild animals; Izimaplasmataceae (5.4\% in wild), p-251-05 (5.3\% in wild), and an unclassified Bacteroidia family (3.5\%). We used ANCOM to statistically test whether there were any families of microbes that were differentially abundant between captive and wild individuals. Twenty-one families were found to be significantly differentially abundant between captive and wild individuals (SI File 2).

At the sequence-level, we found 181 ASVs that were significantly differentially abundant between captive and wild individuals (SI File 3). We next sought to determine whether there were ASVs only detected in captive or wild SHNWs. After filtering feature tables to conservatively account for cross-contamination between samples (see methods), the total number of ASVs in the captive samples went from 2618 (1,292,572 total sequences) to $550(1,107,746)$, and $3096(5,026,870)$ to $642(4,729,528)$ for wild samples. From these ASVs, 449 were found only in captive SHNWs, accounting for $73.7 \%$ of the total abundance, with 541 ASVs being found only in wild SHNWs (71.3\% abundance). One hundred and one ASVs were shared between the captive and wild SHNWs, accounting for 26.3 and $28.7 \%$ of their faecal microbiota abundance, respectively (Fig. 3b). To determine the distribution of these ASVs across the samples, we calculated the core microbiota (i.e. ASVs found in at least N\% of samples) for both between and within captive and wild SHNWs. Six ASVs were found in all samples $(0.5 \%$ of total ASVs), and 31 were found in at least $90 \%$ of all samples (2.8\% of total ASVs) (Table 1). Thirty-seven ASVs were found in all captive samples (6.7\% of total captive ASVs), and 105 were found in at least $90 \%$ of captive samples (19\% of total captive ASVs). Forty-nine ASVs were found in all wild samples $(7.6 \%$ of total wild ASVs), and 193 ASVs were found in at least $90 \%$ of wild samples (30\% of total wild ASVs).

We next used SINA (SILVA Incremental Aligner) to contextualize the most abundant core ASVs (SI Table 3). The most abundant 100 and $90 \%$ core ASVs to all samples were classified as UCG-005 and Treponema, respectively, with a 97.3 and $95.3 \%$ nucleotide identity to the references. The most abundant 100 and $90 \%$ core 


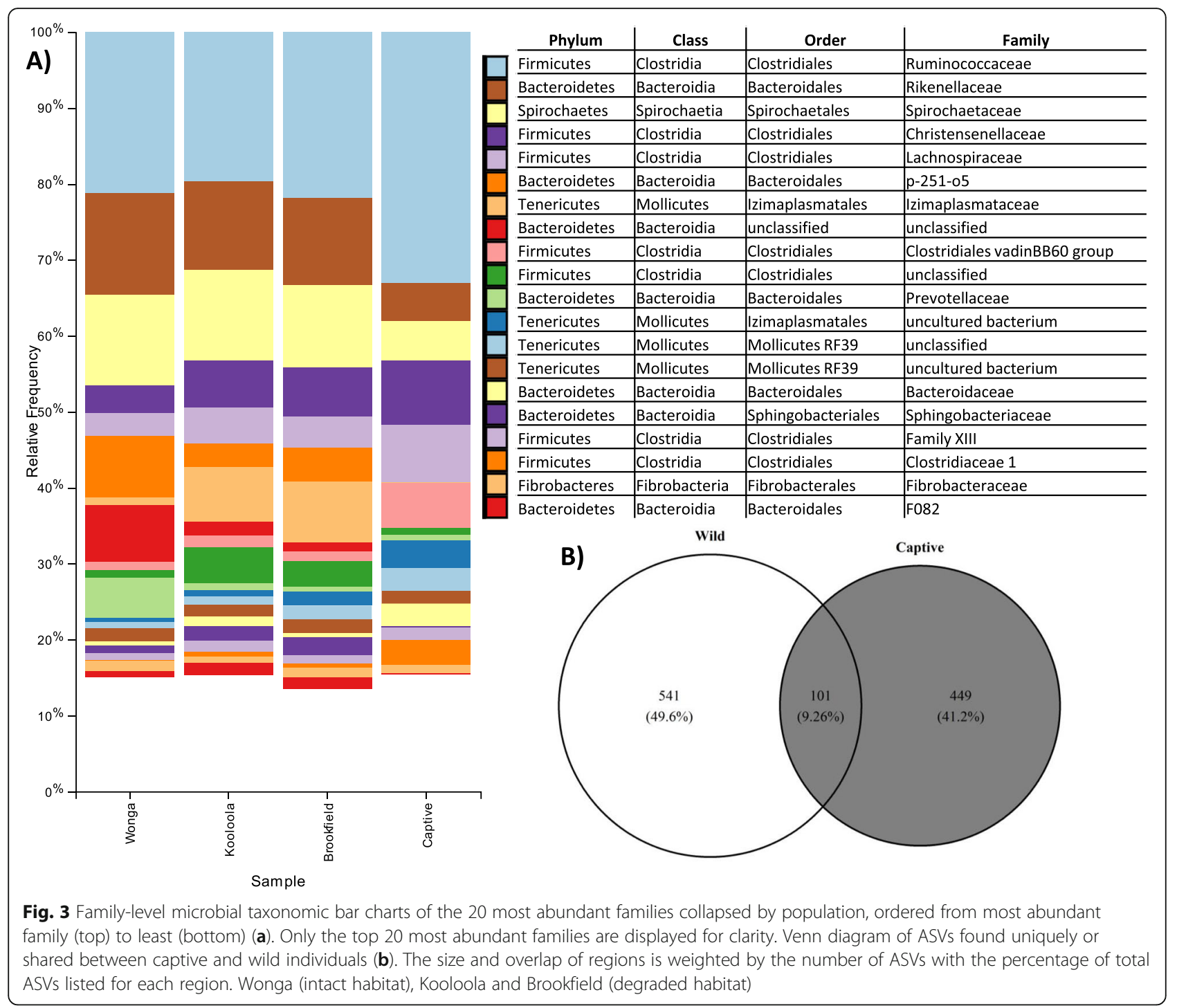

Table 1 Core ASV statistics between and within different SHNW populations

\begin{tabular}{llll}
\hline Population & Captive + Wild & Captive & Wild \\
\hline \# sequences & $5,837,274$ & $1,107,746$ & $4,729,528$ \\
\# ASVs & 1091 & 550 & 642 \\
\# 100\% core ASVs & 6 & 37 & 49 \\
\% of total ASVs & $0.5 \%$ & $6.7 \%$ & $7.6 \%$ \\
\# sequences from 100\% core ASVs & 103,657 & 98,989 & 953,577 \\
\% abundance & $1.8 \%$ & $8.9 \%$ & $20.2 \%$ \\
\# 90\% core ASVs & 31 & 105 & 193 \\
\% of total ASVs & $2.8 \%$ & $19 \%$ & $30 \%$ \\
\# sequences from $90 \%$ core ASVs & $1,073,221$ & 268,413 & $2,983,540$ \\
\% abundance & $18.4 \%$ & $24.2 \%$ & $63.1 \%$ \\
\hline
\end{tabular}




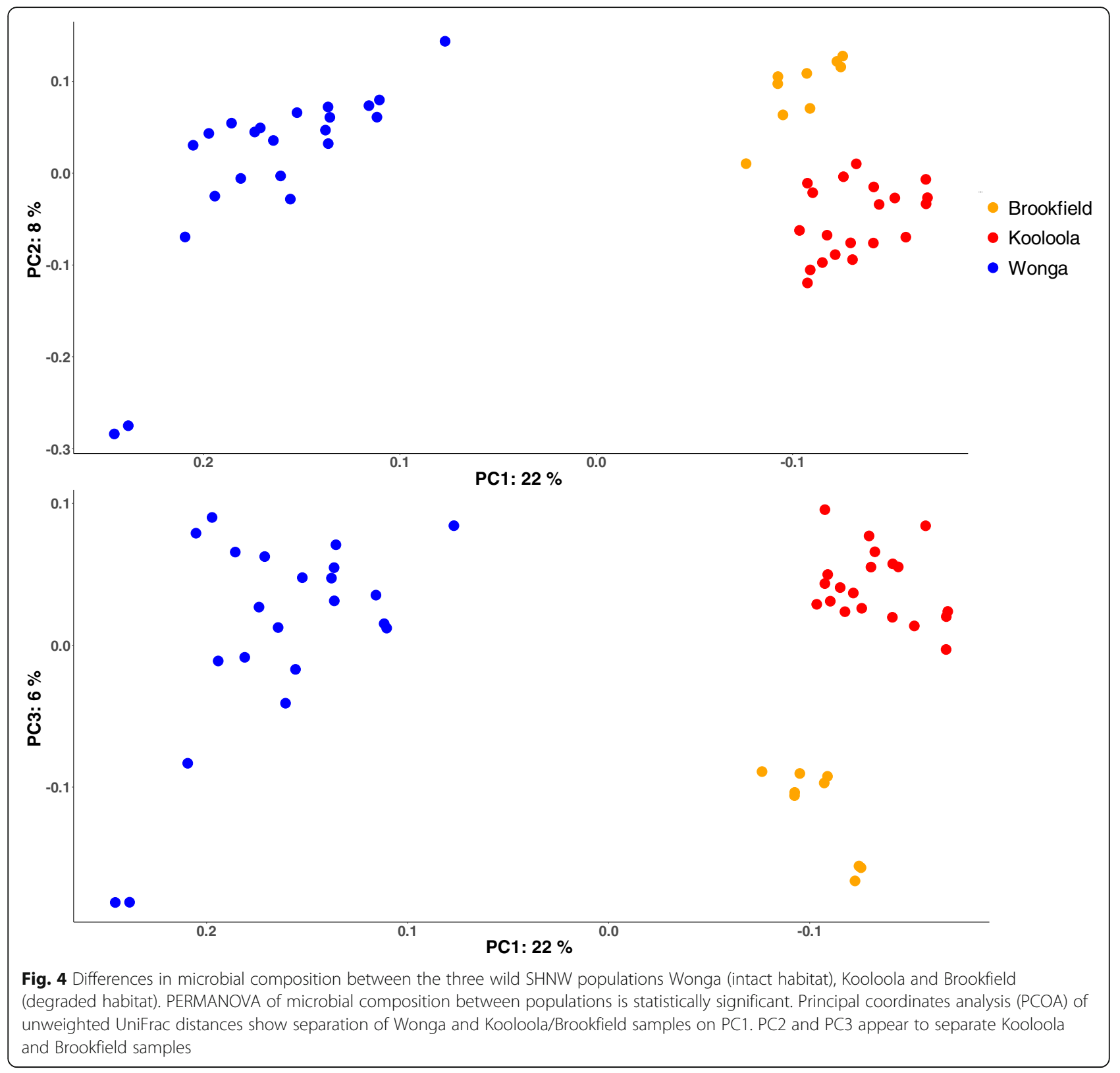

ASV in the captive samples was the same UCG-005 ASV. The most abundant $100 \%$ core ASV in the wild samples was classified to Treponema, with a $90.7 \%$ nucleotide identity to the reference. Finally, the most abundant $90 \%$ core ASV in the wild samples was also the most abundant ASV in the entire dataset, accounting for $5.8 \%$ of the total sequences in wild samples. This ASV was classified only to the order Bacteroidales, with only $86.7 \%$ nucleotide identity to the nearest reference. Overall, these results suggest that captivity has a major influence on SHNW gut microbiota diversity and composition, and that the SHNW gut contains bacteria that are highly divergent from current database references.
Population-level gut microbiota signatures in the SHNW Next, we focused on comparing and contrasting microbial composition between different wild SHNW populations, which include differences in habitat (Wonga has intact native grasses, forbs and chenopods, whereas Kooloola and Brookfield represent degraded pastoral habitat with introduced weed species comprising most of the SHNWs diet).

Samples from Wonga were clearly separated from Brookfield and Kooloola along the first principal coordinate, which explained $22 \%$ of the variation (Fig. 4), and these differences in microbial composition were statistically significant (PERMANOVA of unweighted UniFrac 
distances $p$-values $=0.001$, pseudo- $\mathrm{F}=12.1$ and 7.9 for Wonga vs. Kooloola and Wonga vs. Brookfield, respectively). Samples from Kooloola and Brookfield were also separated from each other across principal coordinates 2 and 3, which explained 8 and $6 \%$ of the variation, respectively, and these differences were also statistically significant (PERMANOVA of unweighted UniFrac distance $p$-value $=0.001$, pseudo- $\mathrm{F}=4.3$ ). These results were also similar when using the weighted Unifrac metric, with the exception of the differences between Brookfield and Kooloola samples (SI Fig. 4).

Using ANCOM, we found 7 families that had differential abundance between wild populations (SI File 4). Streptococcaceae had a higher relative abundance in Kooloola samples, an unclassified RF39 family was only found in Kooloola and Brookfield samples, and Micrococcaceae had a higher relative abundance in Wonga samples. At the sequence level, 14 ASVs were significantly differentially abundant between populations (SI File 5). Between the different SHNW populations, 111 ASVs were found only in Kooloola samples (accounting for $7.6 \%$ total abundance), 79 were found only in Brookfield samples (4\%), 181 ASVs were found only in the
Wonga samples (10.9\%), and 267 were shared in all wild samples (Fig. 5). To test whether these populationspecific ASVs were due to differences in soil microbiota between sampling sites, we ran SourceTracker2 with the soil samples set as sources and the faecal samples set as sinks. We found that soil microbes were not a major component of the SHNW faecal samples (mixing proportions $<0.001$; SI Table 4).

\section{Discussion}

Captive management of wild mammals is typically accompanied by a drastic shift in environment and diet, factors that have been shown to have a large influence on the gut microbiota of mammals. In addition, different populations of the same animal species have been found to harbour unique gut microbial communities, especially across distinct habitats $[14,15]$. These findings spurred us to investigate their relevance to the gut microbiota of the SHNW, a species of conservation significance [24]. We found that captive SHNWs had reduced gut microbiota diversity and a substantially different microbial composition compared to wild SHNWs. We also found population-specific differences in microbial composition

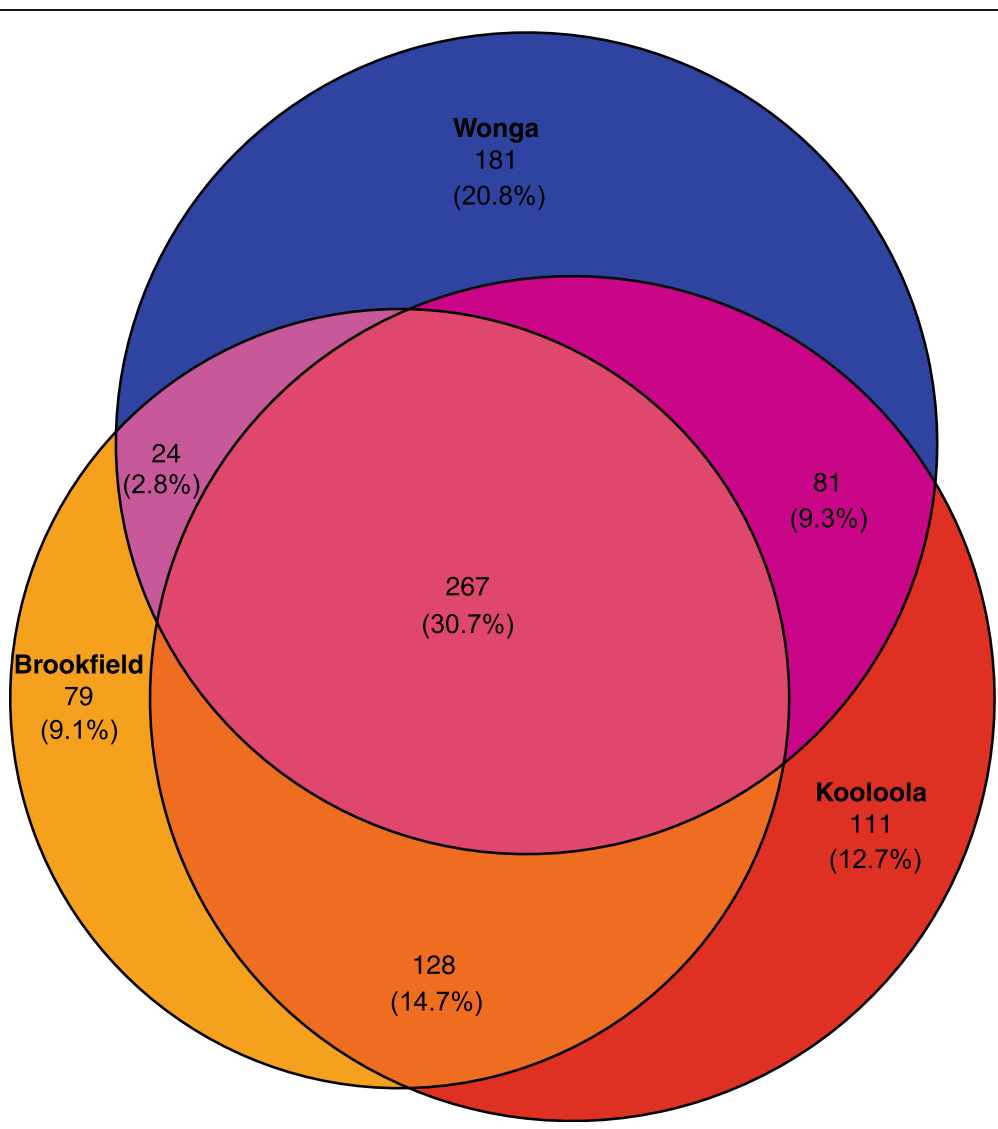

Fig. 5 Venn diagram of ASVs shared and unique between different SHNW populations, with the percentage of total ASVs listed for each region. Wonga (intact habitat), Kooloola and Brookfield (degraded habitats) 
between the three wild SHNW populations surveyed, with the largest differences occurring between SHNWs living in intact versus degraded habitats.

The gut microbiota of mammalian herbivores provides key functions involved in the extraction of nutrients and energy from the diet that would otherwise be unavailable to the host $[52,53]$. This reliance of herbivorous mammals on their gut microbial communities has resulted in physiological and morphological adaptations of the mammalian gastrointestinal tract to enhance the extraction of energy by the gut microbiota, including an increase in length and capacity [54]. The SHNW is a hindgut fermenter with an extremely long and capacious gastrointestinal tract ( 12 times body length) [21]. As the diet of SHNWs largely consists of plant cell walls that are recalcitrant to host enzymes, SCFAs produced through the microbial degradation and fermentation of these compounds in the colon are thought to form the bulk (>60\%) of the daily energy requirements of SHNWs [6]. Previous metagenomic functional profiling of a single captive SHNW found microbial capacity for the degradation of complex plant polysaccharides, including cellulose, xylan, and hemicellulose [55]. While such functions were found in a captive SHNW, we would predict that captivity reduces the diversity of such genes, which could impact the ability of SHNWs to derive energy from wild diets. Disruptions to the gut microbiota of SHNWs could therefore have major negative impacts to their health and fitness.

Our finding that captive SHNWs harboured reduced microbial diversity and substantially different microbial composition compared to wild SHNWs follows the general trend previously observed in other mammalian species [7, 9]. At the sequence level we observed major differences, with only $7.8 \%$ of ASVs being shared between captive and wild SHNWs -- accounting for only 26.3 and $28.7 \%$ of their gut microbiota, respectively. The marked difference in diet could be a major contributing factor, with captive SHNWs in our study being fed a more digestible diet. Such a diet could select for a gut microbiota adapted to simple sugars and starch, with less reliance on complex metabolic pathways involved in the degradation and fermentation of resistant polysaccharides. This has been previously observed in humans, with Italian children consuming a diet containing less fibre and more starches having lower diversity and depleted levels of microbes associated with complex carbohydrate metabolism compared to children living in Burkina Faso who consumed a diet high in fibre [56]. This has also been demonstrated experimentally in laboratory mice, with a loss of microbial diversity being linked to a reduction of dietary fibre [12].

Like many other mammals [57], the SHNW gut microbiota was dominated $(>70 \%)$ by the phyla Firmicutes and Bacteroidetes. We observed substantial differences in the relative abundance of these phyla between captive and wild SHNWs, with captive animals having a mean Firmicutes:Bacteroidetes ratio of 4.8:1 vs. 1.7-0.8:1 for wild populations. This could be due to the differences in availability and types of fibre present in captive and wild diets, as there are species within the Bacteroidetes and Firmicutes phyla that are known to harbour diverse carbohydrate active enzymes [58]. In humans, an increase in Bacteroidetes and decrease in Firmicutes was observed in children consuming larger quantities and diversity of fibre [56]. Mice fed a diet low in fibre also preferentially lost microbes belonging to the phylum Bacteroidetes [12]. Such changes in abundance or loss may result in a reduced capacity to produce SCFAs. Children eating less fibre had significantly lower concentrations of SCFA measured in faeces compared to children on a fibre-rich diet [56]. Barboza \& Hume also found that wild SHNWs produced substantially more SCFAs than captive animals, with wild animals also producing greater quantities of propionate and reduced quantities of butyrate compared to captive held SHNWs [6].

However, caution should be used when interpreting the putative functions of microbes based on their taxonomy, particularly in our dataset, as the microbes of Australian marsupials remain little studied. Many of the microbes classified in this study were distantly related to characterised microbes-the most abundant ASV had only $86.7 \%$ nucleotide identity to the closest reference database sequence. Further genome-centric and biochemical analyses will be required to test the hypothesis that captivity results in a loss of microbial metabolic potential to degrade and ferment complex plant polysaccharides in SHNWs. Overall, our findings suggest that captivity results in a loss of microbial diversity and marked shifts in the gut microbiota composition of SHNWs. Such changes could reduce the energy economy of SHNWs being translocated from captivity to the wild. While the SHNW is only considered "Near-Threatened" at present, its close relative the Northern Hairynosed Wombat (Lasiorhinus krefftii) is listed as critically endangered, with an estimated population size $<300$ [59]. Future captive breeding programs for this species should take precautionary steps to reduce the loss of wild gut microbiota diversity in captivity, such as the feeding of natural diets [13].

We also observed significant differences in microbial composition between the three different wild SHNW populations sampled, with the largest difference being between Wonga (intact habitat) versus Brookfield and Kooloola (degraded habitats). One possible reason for this finding is that differences in floral assemblages between intact/degraded habitats selected for distinct gut microbial compositions that were better adapted to deriving energy 
from the different plant species. Previous work on herbivorous primates identified changes in gut microbial diversity and composition between populations from habitats of differing quality $[14,15]$, with animals from native habitats possessing a greater number of genes associated with SCFA production and hydrogen metabolism [14]. These differences also align with significant differences in body condition score between wombats from Wonga and Kooloola across all cohorts, with animals from Wonga (intact habitat) in significantly better condition, at all times of the year, than those from Kooloola (degraded habitat) (Taggart et al. in preparation). The implications of these findings on breeding, survival of young, and resilience to drought in their semi-arid environment are likely to be significant. Another possible reason for these differences could relate to the presence of toxic plant species in the degraded habitats (Brookfield/Kooloola). These degraded habitats harboured plant species including Potato weed, Ward's weed, and Onion weed, and contain toxic plant defence compounds (eg. pyrrolizidine alkaloids) [31, 60]. Previous work on other herbivorous mammals has demonstrated that the gut microbiota can detoxify plant defence compounds for the host [61-63]. Therefore, some of the gut microbes identified as unique to SHNWs living in degraded habitats could play roles in the detoxification of plant defence compounds. To test these possible factors, shotgun metagenomics could be used to identify genes or pathways associated with the degradation of different plant cell wall components, or the detoxification of plant defence compounds.

Seasonal variation (e.g. temperature, rainfall and food availability) can play a major role in shaping the gut microbiota of animals [64]. As seasons affect plant assemblages in these habitats (e.g. grasses tend to emerge after adequate rainfall, and subside in drier months), sampling the same wombat populations at different times of the year could yield insights into how the gut microbiota is changing or adapting to host diet. The South Australian Murraylands, where this study was undertaken, has a semi-arid environment with most rainfall and associated plant growth occurring in winter and spring followed by long, hot and dry summer and autumn months. In this study, wombat faecal samples from degraded habitats (Kooloola and Brookfield) were collected in March (autumn) and those from the intact habitat (Wonga) were collected 3 months later in mid June (start of winter). However, in 2019 when this study was conducted the region was in drought with little rainfall falling in the first half of the year, and minimal rainfall (5$7 \mathrm{~mm} /$ month) recorded prior to sampling at any of the sites we investigated: Kooloola $14 \mathrm{~mm}$ between Jan March, (BOM, Swan Reach); Brookfield $20 \mathrm{~mm}$ between Jan-March (BOM, Blanchtown); and Wonga $45.6 \mathrm{~mm}$ between Jan - mid June (BOM, Robertstown). As a consequence, rainfall associated vegetation changes were also minimal at these sites. Differences in sampling times in this study are thus unlikely to drive the differences in wombat gut microbiomes observed between degraded and intact landscapes. Finally, host sex has been shown to influence the gut microbiota of vertebrates [65], and while our study could not determine the sex of wild animals, it appears that capitivity and population are more important drivers of the SHNW gut microbiota.

An alternate explanation for the differences in SHNW gut microbiota composition between wild populations is neutral allopatric speciation of microbes with their hosts [66]. As populations separate from each other the opportunity for microbes to be shared between them is reduced. Microbes within each separate population can then continue to acquire mutations and co-speciate with their host, leading to observed differences between populations. Dispersal in SHNWs is thought to be limited and biased toward females [27, 67], with most animals staying within several hundred metres of their burrow of birth. Brookfield and Kooloola are physically closer to each other than to Wonga station. Therefore, the observed differences in gut microbiota composition between wild SHNW populations could be a product of minimal dispersal of animals between the populations, precluding the sharing of microbes between populations. Future host genetic investigations into the population structure and history of SHNWs in South Australia would provide a scaffold for which to test whether the gut microbiota is allopatrically speciating. Additionally, greater sampling of SHNW gut microbiota across their entire distribution (including the Nullarbor, which is $1000 \mathrm{~km}$ west of the populations tested here) will also be needed to test this scenario.

\section{Conclusions}

We found that captivity significantly altered the diversity and composition of the gut microbiota in SHNWs. We also detected population-specific microbial compositions in the gut microbiota of wild SHNWs, which could represent microbial adaptations to the different habitats. How important these microbial differences are to host health and fitness remains to be determined, but could have management implications for SHNWs in the future, such as modifying captive diets or using faecal microbiota transplants to assist with SHNW translocations.

\section{Supplementary Information}

The online version contains supplementary material available at https://doi. org/10.1186/s42523-020-00068-y.

Additional file 1: SI_Figure_1. PCoA plot of unweighted UniFrac distances including the 7 outlier Brookfield samples. 
Additional file 2: SI_Figure_2. Phylum-level taxonomic barplots including the 7 outlier Brookfield samples.

Additional file 3: SI_Figure_3. PCoA plots of weighted UniFrac distances for captive and wild samples.

Additional file 4: SI_Figure_4. PCoA plots of weighted UniFrac distances for the different populations of wild samples.

Additional file 5: SI_Table_1. Pairwise Kruskal-Wallis test results for Faith's phylogenetic diversity metric comparisons between populations.

Additional file 6: SI_Table_2. Pairwise Kruskal-Wallis test results for observed features (richness) metric comparisons between populations.

Additional file 7: SI_Table_3. SINA alignment results of the most abundant core ASVs in SHNWs.

Additional file 8: SI_Table_4. SourceTracker2 results testing the estimated proportion of faecal bacteria coming from soil. https://doi.org/ 10.25909/12979157.

Additional file 9: SI_File_1. QIIME2 qzv file of taxonomic bar plots per sample.

Additional file 10: SI_File_2. QIIME2 qZv file of ANCOM test at family level (captive and wild samples).

Additional file 11: SI File 3. QIIME2 qZV file of ANCOM test at the ASV level (captive and wild samples).

Additional file 12: SI_File_4. QIIME2 qZV file of ANCOM test at family level (between different wild populations).

Additional file 13: SI_File_5. QIIME2 qZv file of ANCOM test at the ASV level (between different wild populations).

\section{Abbreviations}

ASV: Amplicon Sequence Variant; HTS: High-Throughput Sequencing: PCoA: Principal Coordinates Analysis; PCR: Polymerase Chain Reaction; SCFA: Short-Chain Fatty Acid; SHNW: Southern Hairy-nosed Wombat

\section{Acknowledgements}

We would like to acknowledge the help and support of Manfred Heide, who provided captive SHNW faecal samples. We would also like to thank Calvin Heath for his help with the field and lab work.

\section{Authors' contributions}

All authors initiated and conceived the project. DT and RE collected the samples. RE performed the molecular work, bioinformatic and statistical analysis. All authors wrote, edited, and approved the final manuscript.

Authors' information

Not applicable.

\section{Funding}

This project was funded by the Australian Research Council Centre of Excellence for Australian Biodiversity and Heritage (CABAH) and the Schultz Foundation.

\section{Availability of data and materials}

Raw sequencing data are available at the NCBI SRA (BioProject ID PRJNA663974)

All QIIME2, code, analysis files, and R code used to plot figures can be found here: https://github.com/EisenRa/2020_SHNW_Faecal_16S.

All files have also been frozen at the following DOI: https://zenodo.org/ badge/latestdoi/316123393.

\section{Ethics approval and consent to participate}

Not applicable.

\section{Consent for publication}

Not applicable.

\section{Competing interests}

The authors declare that they have no competing interests.

\section{Author details}

School of Biological Sciences, University of Adelaide, Adelaide, South Australia, Australia. ${ }^{2}$ Australian Research Council Centre for Australian Biodiversity and Heritage, University of Adelaide, Adelaide, South Australia, Australia. ${ }^{3}$ Australian Museum Research Institute, 1 William St, Sydney, New South Wales, Australia. ${ }^{4}$ Australian Research Council Centre for Australian Biodiversity and Heritage, University of New South Wales, Sydney, New South Wales, Australia. ${ }^{5}$ School of Animal and Veterinary Sciences (Waite), University of Adelaide, Adelaide, South Australia, Australia. ${ }^{6}$ FAUNA Research Alliance, PO Box 5092, Kahibah, NSW 2290, Australia.

Received: 19 September 2020 Accepted: 15 December 2020

Published online: 06 January 2021

\section{References}

1. Trevelline BK, Fontaine Samantha S, Hartup Barry K, Kohl Kevin D. Conservation biology needs a microbial renaissance: a call for the consideration of host-associated microbiota in wild life management practices. Proc Royal Soc B: Biol Sci. 2019;286:20182448.

2. McFall-Ngai M, Hadfield MG, Bosch TCG, Carey HV, Domazet-Lošo T, Douglas AE, et al. Animals in a bacterial world, a new imperative for the life sciences. PNAS. 2013;110:3229-36.

3. McKenney EA, Koelle K, Dunn RR, Yoder AD. The ecosystem services of animal microbiomes. Mol Ecol. 2018;27:2164-72.

4. Rowland I, Gibson G, Heinken A, Scott K, Swann J, Thiele I, et al. Gut microbiota functions: metabolism of nutrients and other food components. Eur J Nutr. 2018:57:1-24

5. Kohl KD, Weiss RB, Cox J, Dale C, Dearing MD. Gut microbes of mammalian herbivores facilitate intake of plant toxins. Ecol Lett. 2014;17:1238-46.

6. Barboza PS, Hume ID. Hindqut fermentation in the wombats: two marsupial grazers. J Comp Physiol B. 1992;162:561-6.

7. Kohl KD, Skopec MM, Dearing MD. Captivity results in disparate loss of gut microbial diversity in closely related hosts. Conserv Physiol. 2014:2 Available from: https://www.ncbi.nlm.nih.gov/pmc/articles/PMC4806740/. [cited 2018 Dec 5].

8. Clayton JB, Vangay P, Huang H, Ward T, Hillmann BM, Al-Ghalith GA, et al. Captivity humanizes the primate microbiome. PNAS. 2016;113:10376-81.

9. McKenzie VJ, Song SJ, Delsuc F, Prest TL, Oliverio AM, Korpita TM, et al. The effects of captivity on the mammalian gut microbiome. Integr Comp Biol. 2017:57:690-704.

10. Dahlhausen KE, Doroud L, Firl AJ, Polkinghorne A, Eisen JA. Characterization of shifts of koala (Phascolarctos cinereus) intestinal microbial communities associated with antibiotic treatment. PeerJ. 2018;6:e4452.

11. Muegge BD, Kuczynski J, Knights D, Clemente JC, González A, Fontana L, et al. Diet drives convergence in gut microbiome functions across mammalian phylogeny and within humans. Science. 2011:332:970-4.

12. Sonnenburg ED, Smits SA, Tikhonov M, Higginbottom SK, Wingreen NS, Sonnenburg JL. Diet-induced extinctions in the gut microbiota compound over generations. Nature. 2016;529:212-5.

13. Martínez-Mota R, Kohl KD, Orr TJ, Denise DM. Natural diets promote retention of the native gut microbiota in captive rodents. ISME J. 2020;14: 67-78 Nature Publishing Group.

14. Amato KR, Yeoman CJ, Kent A, Righini N, Carbonero F, Estrada A, et al. Habitat degradation impacts black howler monkey (Alouatta pigra) gastrointestinal microbiomes. ISME J. 2013;7:1344-53 Nature Publishing Group.

15. Barelli C, Albanese D, Donati C, Pindo M, Dallago C, Rovero F, et al. Habitat fragmentation is associated to gut microbiota diversity of an endangered primate: implications for conservation. Sci Rep. 2015;5:14862 Nature Publishing Group.

16. Brice KL, Trivedi P, Jeffries TC, Blyton MDJ, Mitchell C, Singh BK, et al. The Koala (Phascolarctos cinereus) faecal microbiome differs with diet in a wild population. PeerJ. 2019:7:e6534

17. Blyton MDJ, Soo RM, Whisson D, Marsh KJ, Pascoe J, Le Pla M, et al. Faecal inoculations alter the gastrointestinal microbiome and allow dietary expansion in a wild specialist herbivore, the koala. Animal Microbiome. 2019:1:6.

18. Taggart DA, PDM T-S. Southern hairy-nosed wombat. In: Van Dyck S, Strahan R, editors. The Mammals of Australia. Australia: New Holland Publishers (Australia) Pty. Ltd; 2008. p. 204-6. 
19. Swinbourne MJ, Taggart DA, Peacock D, Ostendorf B. Historical changes in the distribution of hairy-nosed wombats (Lasiorhinus spp.): a review. Aust Mammal. 2017;39:1-16 CSIRO PUBLISHING.

20. Swinbourne MJ, Taggart DA, Swinbourne AM, Lewis M, Ostendorf B. Using satellite imagery to assess the distribution and abundance of southern hairynosed wombats (Lasiorhinus latifrons). Remote Sens Environ. 2018;211:196-203.

21. Barboza PS, Hume ID. Digestive tract morphology and digestion in the wombats (Marsupialia: Vombatidae). J Comp Physiol B. 1992;162:552-60.

22. Barboza PS. Digestive strategies of the wombats: feed intake, fiber digestion, and digesta passage in two grazing marsupials with hindgut fermentation. Physiol Zool. 1993;66:983-99 [University of Chicago Press, Division of Comparative Physiology and Biochemistry, Society for Integrative and Comparative Biology].

23. Vombatidae WR. Fauna of Australia volume 1B Mammalia. Australia: Australian Government Publishing Service, Canberra, Australian Capita Territory; 1989. p. 755-68.

24. Taggart DA, Finlayson GR, Sparrow EE, Dibben RM, Dibben JA, Campbell EC, et al. Environmental factors influencing hairy-nosed wombat abundance in semi-arid rangelands. J Wildl Manag. 2020;84:921-9.

25. Wells RT. Field observations of the hairy-nosed wombat, Lasiorhinus latifrons (Owen). Wildl Res. 1978:5:299-303 CSIRO PUBLISHING.

26. Wells R, Green B. Aspects of water metabolism in the southern hairy-nosed wombat Lasiorhinus latifrons. Wells RT, Pridmore PA, editors. Wombats. Chipping Norton: Surrey Beatty and Sons Ltd; 1998. p. 61-6.

27. Finlayson GR, Shimmin GA, Temple-Smith PD, Handasyde KA, Taggart DA. Burrow use and ranging behaviour of the southern hairy-nosed wombat (Lasiorhinus latifrons) in the Murraylands, South Australia. J Zool. 2005;265: 189-200 Cambridge University Press.

28. Finlayson GR, White CR, Dibben R, Shimmin GA, Taggart DA. Activity patterns of the southern hairy-nosed wombat (Lasiorhinus latifrons) (Marsupialia : Vombatidae) in the South Australian Murraylands. Aust Mammal. 2010;32:39-46 CSIRO PUBLISHING.

29. Thornett E, Ostendorf B, Taggart DA. Interspecies co-use of southern hairynosed wombat (Lasiorhinus latifrons) burrows. Aust Mammal. 2017;39:20512 CSIRO PUBLISHING.

30. Horsup $A B$, Johnson CN. Northern hairy-nosed wombat, Lasiorhinus krefftii. In: Van Dyck S, Strahan R, editors. The Mammals of Australia. 3rd ed. Sydney: Reed New Holland; 2008. p. 202-4.

31. Camp A, Croxford AE, Ford CS, Baumann U, Clements PR, Hiendleder S, et al. Dual-locus DNA metabarcoding reveals southern hairy-nosed wombats (Lasiorhinus latifrons Owen) have a summer diet dominated by toxic invasive plants. PLoS One. 2020;15:e0229390 Public Library of Science.

32. Song SJ, Amir A, Metcalf JL, Amato KR, Xu ZZ, Humphrey G, et al. Preservation methods differ in fecal microbiome stability, affecting suitability for field studies. mSystems. 2016;1 Available from: https://www.ncbi.nlm.nih. gov/pmc/articles/PMC5069758/. [cited 2018 May 24].

33. Eisenhofer R, Minich JJ, Marotz C, Cooper A, Knight R, Weyrich LS. Contamination in low microbial biomass microbiome studies: issues and recommendations. Trends Microbiol. 2019;27:105-17.

34. Caporaso JG, Lauber CL, Walters WA, Berg-Lyons D, Lozupone CA, Turnbaugh PJ, et al. Global patterns of 165 rRNA diversity at a depth of millions of sequences per sample. PNAS. 2011;108:4516-22.

35. Marotz C, Sharma A, Humphrey G, Gottel N, Daum C, Gilbert JA, et al. Triplicate PCR reactions for 165 rRNA gene amplicon sequencing are unnecessary. BioTechniques. 2019; Available from: https://www.futurescience.com/doi/full/10.2144/btn-2018-0192. [cited 2019 May 28].

36. Thompson LR, Sanders JG, McDonald D, Amir A, Ladau J, Locey KJ, et al. A communal catalogue reveals Earth's multiscale microbial diversity. Nature. 2017;551:457-63.

37. Bolyen E, Rideout JR, Dillon MR, Bokulich NA, Abnet CC, Al-Ghalith GA, et al. Reproducible, interactive, scalable and extensible microbiome data science using QIIME 2. Nat Biotechnol. 2019;37:852-7.

38. Amir A, McDonald D, Navas-Molina JA, Kopylova E, Morton JT, Xu ZZ, et al. Deblur rapidly resolves single-nucleotide community sequence patterns. mSystems. 2017;2:e00191-16

39. Quast C, Pruesse E, Yilmaz P, Gerken J, Schweer T, Yarza P, et al. The SILVA ribosomal RNA gene database project: improved data processing and webbased tools. Nucleic Acids Res. 2013;41:D590-6.

40. Bokulich NA, Kaehler BD, Rideout JR, Dillon M, Bolyen E, Knight R, et al. Optimizing taxonomic classification of marker-gene amplicon sequences with QIIME 2's q2-feature-classifier plugin. Microbiome. 2018;6:90.
41. Janssen S, McDonald D, Gonzalez A, Navas-Molina JA, Jiang L, Xu ZZ, et al. Phylogenetic placement of exact amplicon sequences improves associations with clinical information. mSystems. 2018;3:e00021-18.

42. Faith DP. Conservation evaluation and phylogenetic diversity. Biol Conserv. 1992;61:1-10.

43. Lozupone CA, Hamady M, Kelley ST, Knight R. Quantitative and qualitative beta diversity measures lead to different insights into factors that structure microbial communities. Appl Environ Microbiol. 2007;73:1576-85.

44. Lozupone C, Knight R. UniFrac: a new phylogenetic method for comparing microbial communities. Appl Environ Microbiol. 2005;71:8228-35.

45. Mandal S, Treuren WW, White RA, Eggesbø M, Knight R, Peddada SD. Analysis of composition of microbiomes: a novel method for studying microbial composition. Microbial Ecol Health Dis. 2015;26 Available from: http://www.microbecolhealthdis.net/index.php/mehd/article/view/27663. [cited 2015 Dec 3].

46. Pruesse E, Peplies J, Glöckner FO. SINA: accurate high-throughput multiple sequence alignment of ribosomal RNA genes. Bioinformatics. 2012;28:1823-9.

47. Knights D, Kuczynski J, Charlson ES, Zaneveld J, Mozer MC, Collman RG, et al. Bayesian community-wide culture-independent microbial source tracking. Nat Methods. 2011:8:761-3.

48. McMurdie PJ, Holmes S. phyloseq: An R package for reproducible interactive analysis and graphics of microbiome census data. PLoS One. 2013;8:e61217 Public Library of Science.

49. Wickham H. ggplot2: Elegant Graphics for Data Analysis. New York: Springer-Verlag; 2016. Available from: https://ggplot2.tidyverse.org.

50. RStudio Team. RStudio: Integrated Development for R. Boston: RStudio, Inc; 2015. Available from: http://www.rstudio.com/.

51. Minich JJ, Sanders JG, Amir A, Humphrey G, Gilbert JA, Knight R. Quantifying and understanding well-to-well contamination in microbiome research. mSystems. 2019;4 Available from: https://msystems.asm.org/content/4/4/ e00186-19. American Society for Microbiology Journals. [cited 2020 Apr 15].

52. Bergman EN. Energy contributions of volatile fatty acids from the gastrointestinal tract in various species. Physiol Rev. 1990;70:567-90 American Physiological Society.

53. Flint HJ, Bayer EA, Rincon MT, Lamed R, White BA. Polysaccharide utilization by gut bacteria: potential for new insights from genomic analysis. Nat Rev Microbiol. 2008;6:121-31 Nature Publishing Group.

54. Hume ID, Warner ACl. Evolution of microbial digestion in mammals. In: Ruckebusch $Y$, Thivend $P$, editors. Digestive physiology and metabolism in ruminants: proceedings of the 5 th international symposium on ruminant physiology, held at Clermont — Ferrand, on 3rd-7th September, 1979. Dordrecht: Springer Netherlands; 1980. p. 665-84. Available from: https:// doi.org/10.1007/978-94-011-8067-2_32. [cited 2020 Sep 2].

55. Shiffman ME, Soo RM, Dennis PG, Morrison M, Tyson GW, Hugenholtz P. Gene and genome-centric analyses of koala and wombat fecal microbiomes point to metabolic specialization for Eucalyptus digestion. PeerJ. 2017:5:e4075.

56. Filippo CD, Cavalieri D, Paola MD, Ramazzotti M, Poullet JB, Massart S, et al. Impact of diet in shaping gut microbiota revealed by a comparative study in children from Europe and rural Africa. PNAS. 2010;107:14691-6.

57. Ley RE, Hamady M, Lozupone C, Turnbaugh PJ, Ramey RR, Bircher JS, et al. Evolution of mammals and their gut microbes. Science. 2008;320:1647-51.

58. Flint HJ, Scott KP, Duncan SH, Louis P, Forano E. Microbial degradation of complex carbohydrates in the gut. Gut Microbes. 2012;3:289-306.

59. Horsup AB, Austin JJ, Fewster RM, Hansen BD, Harper DE, Molyneux JA, et al. Demographic trends and reproductive patterns in the northern hairynosed wombat (Lasiorhinus krefftii) at Epping Forest National Park (Scientific), central Queensland. Aust Mammal. 2020; Available from: https:// www.publish.csiro.au/am/AM20030. CSIRO PUBLISHING; [cited 2020 Sep 2].

60. Woolford L, Fletcher MT, Boardman WSJ. Suspected pyrrolizidine alkaloid hepatotoxicosis in wild southern hairy-nosed wombats (Lasiorhinus latifrons). J Agric Food Chem. 2014;62:7413-8.

61. Allison MJ, Hammond AC, Jones RJ. Detection of ruminal bacteria that degrade toxic dihydroxypyridine compounds produced from mimosine. Appl Environ Microbiol. 1990;56:590-4.

62. Sundset MA, Barboza PS, Green TK, Folkow LP, Blix AS, Mathiesen SD. Microbial degradation of usnic acid in the reindeer rumen. Naturwissenschaften. 2010;97:273-8.

63. Kohl KD, Dearing MD. The woodrat gut microbiota as an experimental system for understanding microbial metabolism of dietary toxins. Front 
Microbiol. 2016;7 Available from: https://www.frontiersin.org/articles/10.33 89/fmicb.2016.01165/full. [cited 2019 Oct 14].

64. Maurice CF, Knowles CL, Ladau J, Pollard KS, Fenton A, Pedersen AB, et al. Marked seasonal variation in the wild mouse gut microbiota. ISME J. 2015;9: 2423-34.

65. Bolnick DI, Snowberg LK, Hirsch PE, Lauber CL, Org E, Parks B, et al. Individual diet has sex-dependent effects on vertebrate gut microbiota. Nat Commun. 2014;5:4500 Nature Publishing Group.

66. Groussin M, Mazel F, Alm EJ. Co-evolution and co-speciation of host-gut Bacteria systems. Cell Host Microbe. 2020;28:12-22.

67. Walker FM, Taylor AC, Sunnucks P. Female dispersal and male kinship-based association in southern hairy-nosed wombats (Lasiorhinus latifrons). Mol Ecol. 2008;17:1361-74

\section{Publisher's Note}

Springer Nature remains neutral with regard to jurisdictional claims in published maps and institutional affiliations.

Ready to submit your research? Choose BMC and benefit from:

- fast, convenient online submission

- thorough peer review by experienced researchers in your field

- rapid publication on acceptance

- support for research data, including large and complex data types

- gold Open Access which fosters wider collaboration and increased citations

- maximum visibility for your research: over $100 \mathrm{M}$ website views per year

At $\mathrm{BMC}$, research is always in progress.

Learn more biomedcentral.com/submissions 